\title{
Revisão sistemática qualitativa sobre avaliações de serviços em saúde mental na perspectiva dos usuários*
}

\section{Éllen Cristina Ricci ${ }^{1,2}$ \\ iD https://orcid.org/0000-0003-3471-1479 \\ Mariana Barbosa Pereira ${ }^{1,2}$ \\ (D) https://orcid.org/0000-0003-3276-9407 \\ Leidy Janeth Erazo ${ }^{1,2}$ \\ (iD) https://orcid.org/0000-0003-3715-7864 \\ Rosana Teresa Onocko-Campos ${ }^{1}$ \\ iD https://orcid.org/0000-0003-0469-5447 \\ Erotildes Maria Leal ${ }^{3}$ \\ (iD) https://orcid.org/0000-0002-8468-4571}

\footnotetext{
* Artigo extraído da tese de doutorado "Avaliação de serviços da saúde mental em relação ao recovery/recuperação sob a perspectiva dos usuários: tradução, adaptação transcultural e validação do instrumento Recovery Self-Assessment (RSA-R).", apresentada à Faculdade de Ciências Médicas, Unicamp 2019, Campinas, SP, Brasil.

${ }^{1}$ Universidade Estadual de Campinas, Faculdade de Ciências Médicas, Campinas, SP, Brasil.

2 Bolsista da Coordenação de Aperfeiçoamento de Pessoal de Nível Superior (CAPES), Brasil.

${ }^{3}$ Universidade Federal do Rio de Janeiro, Faculdade de Medicina, Rio de Janeiro, RJ, Brasil.
}

Objetivo: identificar publicações nacionais brasileiras e internacionais na área de saúde mental sobre avaliação de serviços, destacando as formas de participação dos usuários. Método: trata-se de uma revisão sistemática qualitativa da literatura, que seguiu as orientações do PRISMA e utilizou duas plataformas internacionais que agregam um número significativo de bases de dados, com os descritores (saúde mental e avaliação) no período de 2004 a 2016. Resultados: levantou-se 4.735 artigos completos; tendo elegibilidade final de 137 artigos para leitura integral, sendo 44 incluídos na análise final. Percebe-se que há pesquisas sobre avaliação de serviços de saúde mental com a participação dos usuários no Brasil, mas ainda em número menor em comparação com Inglaterra, Austrália e EUA. Conclusão: a forma de participação dos usuários nas avaliações dos serviços, tanto no Brasil quanto em outros países, ainda é um desafio. Há pouco protagonismo dos usuários. No Brasil, coloca-se o desafio ainda de estruturar avaliações sistemáticas dos serviços públicos, com multiplicidade de métodos avaliativos e envolvendo todos os atores.

Descritores: Avaliação em Saúde; Saúde Mental; Revisão Sistemática; Participação do Paciente.

\section{Como citar este artigo}

Ricci EC, Pereira MB, Erazo LJ, Onocko-Campo RT, Leal EM. Quatitative systematic review of mental health services from the users' perspective. SMAD, Rev Eletrônica Saúde Mental Álcool Drog. 2020;16(2):1-12. doi: https://dx.doi.org/10.11606/issn.1806-6976.smad.2020.159559 


\title{
Quatitative systematic review of mental health services from the users' perspective
}

\begin{abstract}
Objective: to identify, based on qualitative systematic literature review, Brazilian national and international publications in mental health area, addressing service's evaluation on their users perspective. Method: a qualitative systematic literature review, which followed PRISMA orientations and criteria. For that, two international platforms that include a significant number of databases were used, with "mental health" and "evaluation" descriptors and 2004 to 2016 period. Results: 4,735 full articles were initially collected, with final eligibility of 137 for integral text reading, of which 44 were included in the final analysis. Research studies on mental health services evaluation with users' participation are present in Brazil, but still in reduced number when compared to countries like England, Australia and USA. Conclusion: in Brazil, as well as in other countries, the ways by which users participate in services evaluations are still a challenge. Mental health service users' protagonist is insufficient. In Brazil, there is also a challenge to structure public services systematic evaluations, incorporating diversity of evaluative methods and stakeholders' participation.
\end{abstract}

Descriptors: Health Evaluation; Mental Health; Systematic Review; Patient Participation.

\section{Revisión sistemática cualitativa sobre evaluación de servicios de salud mental desde la perspectiva de los usuarios}

Objetivo: identificar publicaciones nacionales brasileñas e internacionales en el área de salud mental sobre evaluación de servicios, con énfasis en las formas de participación de los usuarios. Método: este estudio es una revisión sistemática cualitativa de la literatura, que siguió las recomendaciones de PRISMA y utilizó dos plataformas internacionales que incluyen un número significativo de bases de datos, con los descriptores (salud mental y evaluación) en el periodo de 2004 a 2016. Resultados: se identificaron 4.735 artículos completos; siendo elegidos 137 para lectura completa, al final, se incluyeron 44 en el análisis. Los resultados sugieren que en Brasil hay investigaciones sobre la evaluación de servicios de salud mental que cuentan con la participación de usuarios, pero el número es menor en comparación con Inglaterra, Australia y Estados Unidos. Conclusión: la forma en que los usuarios participan en las evaluaciones de servicios, tanto en Brasil como en otros países, sigue siendo un desafío, hay poco protagonismo de ellos. Brasil, además, tiene el desafío de estructurar evaluaciones sistemáticas de los servicios públicos, con una multiplicidad de métodos de evaluación que involucren a todos los actores.

Descriptores: Evaluación en Salud; Salud Mental; Revisión Sistemática; Participación del Paciente. 


\section{Introdução}

Os projetos de reforma psiquiátrica em diferentes países emergiram no contexto de crítica às instituições asilares após a segunda guerra mundial. Estas foram criticadas devido a sua baixa eficácia terapêutica, aos altos custos de manutenção, a violência com que tratam as pessoas e a exclusão social que geram(1-2).

Esse processo resultou em iniciativas que visam à humanização dos hospitais psiquiátricos, reflexões sobre a qualidade do tratamento e a criação de serviços de saúde mental na comunidade(3). No Brasil esse processo é chamado de Reforma Psiquiátrica Brasileira, que engloba e articula os campos técnico assistencial, político, jurídico, conceitual e sociocultural(4).

Contudo, apesar dos quase 30 anos de implantação dos serviços de saúde mental comunitários no Brasil e 17 anos da Lei 10.216, que dispões sobre os direitos e proteção das pessoas com transtorno mental, que sistematizou, organizou e implantou de forma sistemática os serviços ${ }^{(5-6)}$, o país ainda carece de indicadores de avaliação de $\operatorname{serviços}^{(7)}$.

Há uma escassez de estudos brasileiros com métodos mistos que descrevem processos avaliativos e de resultados, que contemplam a Rede de Atenção Psicossocial (RAPS) principalmente na perspectiva dos usuários ${ }^{(8)}$. No Brasil, o campo da avaliação é incipiente em geral e na saúde mental possui particularidades que tornam o consenso em torno de parâmetros avaliativos um desafio metodológico constante ${ }^{(7-8)}$.

A Organização Mundial de Saúde ${ }^{(9-10)}$ tem produzido informes técnicos e análises para subsidiar objetivos comuns para as políticas setoriais de saúde mental, buscando estabelecer consensos e criar indicadores. Há uma tendência mundial de incorporação dos usuários, a partir da sua experiência de adoecimento e das relações com os espaços de tratamento, nos processos avaliativos dos serviços e de assistência que estes têm prestado.

Entendemos que a participação dos usuários em avaliações sobre os serviços de saúde é fundamental para a obtenção de resultados que os representem, além de influenciar políticas públicas que sejam efetivas e funcionais para o grupo de pessoas atendidas, valorizando o conhecimento que parte da experiência vivida, pensando formas e estratégias de potencializar o diálogo(11).

Portanto, o desafio atual é avaliar a rede de serviços territoriais e a orientação ao tratamento, que valorize a experiência das pessoas no cotidiano e seus processos de recuperação, pois o desafio dos novos serviços é trazer o usuário para o centro da cena do seu tratamento ${ }^{(12-14)}$.

Assim, os resultados encontrados por dois estudos ${ }^{(7-8)}$ sugerem que no Brasil, são mais escassos os trabalhos que se propõem a avaliar serviços de saúde mental na perspectiva e com a participação de seus usuários, quando comparados com o cenário internacional(7-8).
Nosso interesse neste momento foi revelar como esse fenômeno se comporta tanto no cenário nacional como internacional, através de uma revisão sistemática qualitativa sobre a participação dos usuários de serviços de saúde mental na avaliação destes. Para o efeito, levantamos as seguintes questões:

1. Qual é o cenário nacional e internacional de avaliação de serviços em saúde mental com a participação dos usuários do sistema? e;

2. Qual é a forma de participação dos usuários durante o processo de avaliação dos serviços de saúde mental?

O objetivo deste trabalho foi identificar as publicações nacionais brasileiras e internacionais na área da saúde mental, abordando a avaliação de serviços, destacando as formas de participação dos usuários.

\section{Método}

Para alcançar o objetivo proposto neste trabalho realizamos uma revisão sistemática qualitativa seguindo as orientações organizativas de revisões PRISMA(15-16). A revisão bibliográfica sistemática qualitativa prevê que se faça uma síntese de estudos contendo objetivos, materiais e métodos claramente explicados e conduzidos de acordo com um método explícito, reprodutível e rigorosos para identificar textos, fazer avaliação crítica e sintetizar estudos relevantes ${ }^{(17)}$.

Utilizamos duas plataformas internacionais que contemplam número significativo de bases de dados a fim de nos aproximarmos do maior número de produções na área.

A primeira plataforma utilizada foi a Biblioteca Virtual de Saúde - BVS. A escolha dessa fonte deve-se ao seu amplo e consolidado uso no campo da saúde, especialmente no Brasil e na maioria dos países latino americanos. As palavras-chave utilizadas foram: AVALIAÇÃO e SAÚDE MENTAL e os filtros: textos completos disponíveis, país de afiliação Brasil e o período de 2004 a 2016.

Para a revisão internacional a plataforma utilizada foi a Web of Science, em função de seu perfil interdisciplinar, amplitude do escopo de indexação e é assinado pela Unicamp, universidade onde esta revisão ocorreu. As seguintes palavras-chave foram empregadas: EVALUATION and MENTAL HEALTH. Usando os filtros: texto completo disponíveis, idiomas inglês, português e espanhol e ano de publicação de 2004 a 2016.

Formulamos os seguintes critérios de inclusão de textos: avaliação de serviço ou programa especializado em saúde mental feita por usuários, a partir de diferentes aspectos como satisfação com o serviço, narrativas de experiências para fins avaliativos, participação na construção do processo de avaliação como entrevistador e métodos de análise. 
Os critérios de exclusão de textos foram: serviços que não eram exclusivos de saúde mental, serviços de saúde mental infantojuvenil, intervenções pontuais que não avaliavam o serviço como um todo, validação de instrumentos, usuários não participavam da avaliação e outros grupos de interesse que não incluíam os usuários.

Após a primeira etapa desta revisão, montamos a equipe de análise dos dados, composta por três pesquisadoras da área de saúde mental e saúde coletiva. Isso se faz necessário durante o processo de seleção, exclusão e análise do conteúdo dos textos selecionados, para evitar viés de análise, visto que os dados selecionados passaram por dupla avaliação entre membros diferentes da equipe para serem elegíveis ou excluídos. Caso houvesse divergência entre dois membros em relação à inclusão ou não do texto, um terceiro corretor verificava e discutia com a equipe até chegarem a um consenso(16,18-19).

A partir das plataformas, encontramos um total de 4.735 artigos completos. Percebe-se logo na análise em relação ao título, que os avaliadores selecionaram 394 artigos dos 4735. Isso ocorreu, principalmente, porque os títulos já descreviam os critérios de exclusão elencados anteriormente por não contribuírem com o objetivo deste estudo. A maioria dos artigos eram sobre processos avaliativos de medicação, algumas práticas pontuais e qualidade de vida após breves intervenções, sem caracterizar avaliação de serviços.
Também encontramos grande quantidade de pesquisas que ouviram apenas uma parte dos interessados, em sua maioria os trabalhadores do serviços.

Os 394 textos selecionados foram sistematizados a partir das seguintes informações: nome do avaliador, avaliação de inclusão ou exclusão, título do artigo, ano, palavras-chave, autores, métodos utilizados em relação a participação dos usuários, tipo de serviço avaliado, país que foi feita a pesquisa, técnicas utilizadas, resultados encontrados, observações relevantes e endereço eletrônico do texto.

Em seguida, a leitura dos resumos reduziu nossa amostra para 137 artigos. Por esta foi possível identificar artigos que apenas descreviam e avaliavam parte das boas práticas incorporadas aos serviços e tratamentos dispensados. Foi nessa etapa que a maioria dos artigos brasileiros e dos Estados Unidos foram excluídos, por não contemplarem os objetivos desta revisão.

Mantendo a metodologia, duas avaliadoras analisaram os 137 artigos na íntegra e emitiram seu julgamento sobre inserção ou não no processo de análise final. Nesta etapa, não houve divergências na maior parte dos artigos, sendo que apenas 3 deles precisaram da terceira avaliação. Assim chegamos aos 44 artigos que iluminam os objetivos do presente trabalho.

A Figura 1 ilustra no formato de fluxograma as etapas e achados numéricos da revisão bibliográfica:

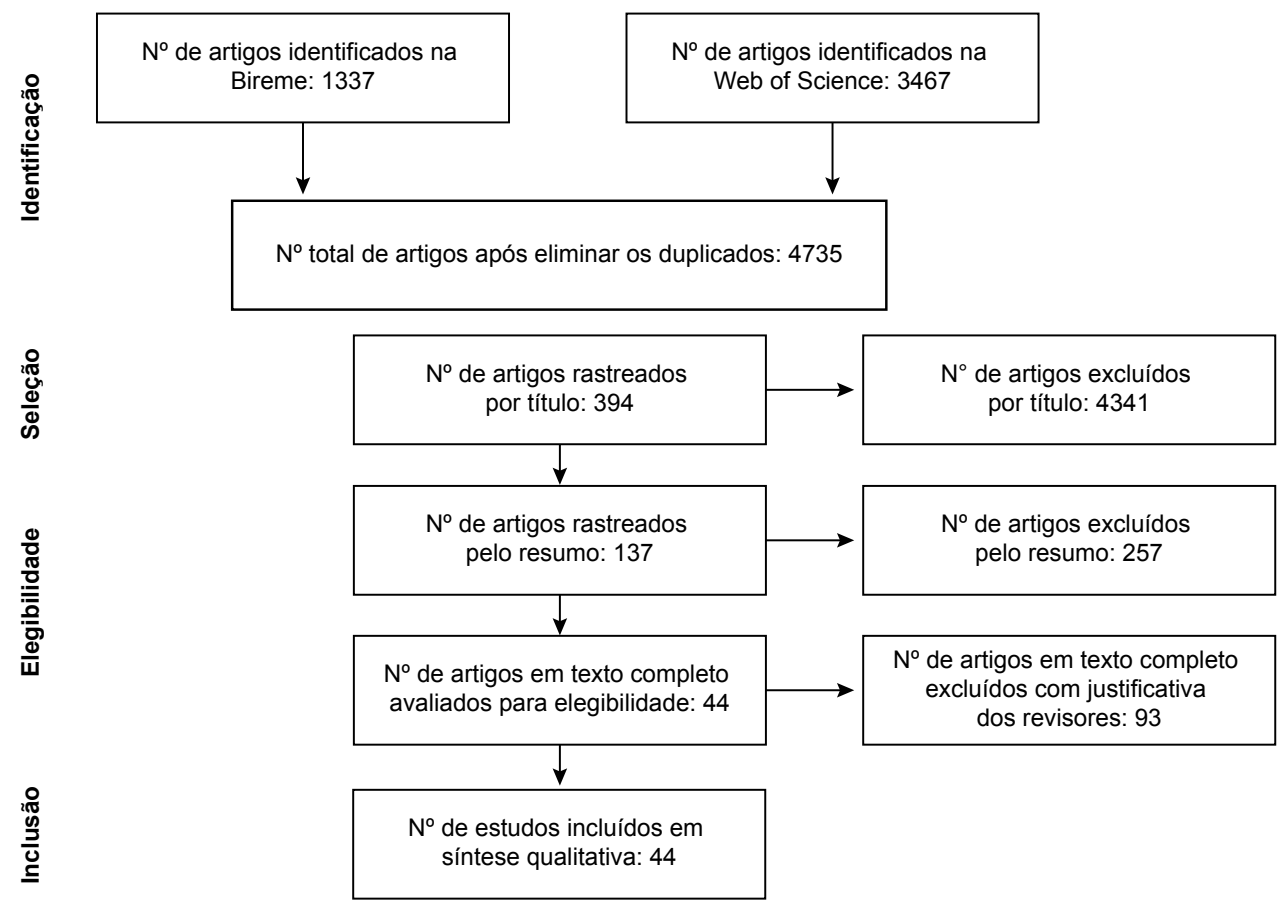

Figura 1- Fluxograma da revisão bibliográfica

Sabemos que no contexto brasileiro é incipiente a participação de usuários como colaboradores e líderes em avaliação e pesquisas, e internacionalmente apenas alguns países têm adotado tais práticas ${ }^{(11,20)}$. Portanto, a partir da literatura estudada, construímos categorias para analisarmos os métodos utilizados em relação à participação dos usuários considerando quatro possibilidades subdivididas da seguinte maneira(11,20):

A1: a perspectiva do usuário é tomada em 'terceira pessoa'; os usuários são assuntos da pesquisa e os 
métodos aplicados são principalmente questionários e outros instrumentos fechados;

A2: a perspectiva do usuário é tomada em 'primeira pessoa'; entrevistas e ou narrativas de experiências do usuário com os serviços;

B1: perspectiva do usuário com sua participação como entrevistador e/ou avaliador em parte do processo conjuntamente com outros avaliadores não usuários;

B2: perspectiva do usuário com sua participação em todas as fases da avaliação, na construção do método e questão a ser avaliada que pode ou não contar com outros atores;

Sendo assim, os artigos selecionados para análise foram tabulados seguindo as categorias acima descritas. Durante todo o percurso utilizamos o programa Excel ${ }^{\circledR}$ para organizações e tabulações dos achados e síntese da amostra e o programa Mendeley ${ }^{\circledR}$ foi utilizado como ferramenta de apoio e organização do material final.

\section{Resultados e Discussão}

Apresentamos na Tabela 1 a distribuições dos 44 artigos selecionados para a análise final por ano de publicação, país de estudo, Tipo de serviço e método de Participação dos Usuários.

Percebe-se que não há uma distribuição homogênea e tão pouco crescente ao longo dos anos, pois temos em 2009 o maior número de artigos encontrados, seguidos pelos anos de 2006 e 2008, tendo 21 artigos produzidos nestes três períodos.

Em relação aos países que apresentam maior produção de artigos sobre avaliação de serviços que incorporam os usuários no processo, destacamos a Inglaterra, Austrália, Estados Unidos seguidos do Brasil e Canadá.

Vale destacar que a Inglaterra, a Austrália e o Canadá são países de cultura avaliativa de serviços, com sistemas públicos nacionais de saúde, que vão além dos dados epidemiológicos e relatórios públicos de gestão, assim dedicam parte dos estudos de avaliação na perspectiva dos usuários/clientes do sistema(11,14). Pesquisas de satisfação sobre o acesso, tratamento e condições dos serviços são comuns, utilizando métodos e ferramentas participativas que contemplam os diferentes atores envolvidos.

Já os EUA têm tradição de avaliação de programas financiados pelo Estado, visto que boa parte da saúde mental depende deste tipo de financiamento para atender a população e não há um sistema nacional único público(11,14). Excluímos diversos artigos que se limitavam apenas a uma prática, avaliação de respostas medicamentosas, ou a um público específico identificado pelo seu diagnóstico recebendo um tratamento pontual.
Tabela 1 - Distribuição dos artigos por frequências - ano de publicação, país, serviço e método de participação dos usuários

\begin{tabular}{|c|c|c|}
\hline & $\mathbf{n}$ & $\%$ \\
\hline \multicolumn{3}{|l|}{ Ano de publicação } \\
\hline 2004 & 2 & 4,5 \\
\hline 2005 & 1 & 2,3 \\
\hline 2006 & 7 & 15,9 \\
\hline 2007 & 2 & 4,5 \\
\hline 2008 & 6 & 13,6 \\
\hline 2009 & 8 & 18,2 \\
\hline 2010 & 5 & 11,4 \\
\hline 2011 & 2 & 4,5 \\
\hline 2012 & 5 & 11,4 \\
\hline 2013 & 2 & 4,5 \\
\hline 2014 & 3 & 6,8 \\
\hline 2015 & 1 & 2,3 \\
\hline \multicolumn{3}{|l|}{ País de Estudo } \\
\hline Alemanha & 1 & 2,3 \\
\hline Austrália & 8 & 18,2 \\
\hline Brasil & 6 & 13,6 \\
\hline Canada & 4 & 9,1 \\
\hline EUA & 6 & 13,6 \\
\hline Finlândia & 1 & 2,3 \\
\hline Inglaterra & 9 & 20,5 \\
\hline Irlanda & 2 & 4,5 \\
\hline Israel & 2 & 4,5 \\
\hline Itália & 2 & 4,5 \\
\hline Suécia & 3 & 6,8 \\
\hline \multicolumn{3}{|l|}{ Tipo de Serviço } \\
\hline APS & 8 & 18,2 \\
\hline Hospital & 9 & 20,5 \\
\hline Rede de Serviços & 2 & 4,5 \\
\hline Serviços especializados & 25 & 56,8 \\
\hline \multicolumn{3}{|c|}{ Método de participação do Usuário } \\
\hline A1 & 18 & 40,9 \\
\hline A2 & 16 & 36,4 \\
\hline B1 & 6 & 13,6 \\
\hline Mais de um método & 4 & 9,1 \\
\hline
\end{tabular}

O Brasil aparece com seis artigos, o que comparado aos países anteriormente citados, nos parece um bom resultado, pois as produções de avaliação geral dos serviços consideram a importância dos usuários neste processo. A avaliação de serviços no Brasil ainda é um grande desafio(14,21). Sabemos que há um grande esforço de pesquisadores brasileiros em criar instrumentos, técnicas, indicadores e guias de boas práticas para avaliação global dos serviços com a participação dos usuários e outros atores, mas os resultados práticos de avaliações sistematizadas ainda não estão no formato de artigos indexados nas bases estudadas.

Como esperávamos, há um grande investimento em entender como usuários e outros atores avaliam os serviços especializados ( 25 dos 44 artigos encontrados), em detrimento aos hospitais (hospitais psiquiátricos, universitários e enfermarias), visto o movimento internacional, que inclui o Brasil, de reformulação do modelo assistencial em saúde mental.

Serviços especializados estão relacionados aos tratamentos de pessoas com transtorno mental grave, álcool e outras drogas, de moradia ou população 
específica (ex. veteranos de guerra nos Estados Unidos contam com uma rede exclusiva). Na Atenção Primária incorporam os trabalhos de prevenção e teleatendimentos. Vale destacar que encontramos dois artigos que avaliavam dois ou mais tipos de serviços concomitantemente (rede de serviços) e incluíam os usuários.

A maioria dos achados se concentrou nas categorias A1 (18 artigos) e A2 (16 artigos) de avaliação. Os artigos em A1 se caracterizavam por entrevistas, questionários que buscavam a avaliação mais objetiva dos serviços, considerando pouco ou nada dos aspectos subjetivos dos usuários. Os que utilizam instrumentos já validados são mais expressivos em experiências internacionais.

A2 trouxe mais entrevistas de opinião abertas, experiências e avaliações que destacavam a experiência vivida por eles e o modo como avaliavam o processo. No que diz respeito à abordagem metodológica pela qual os usuários do serviço participam como sujeitos pesquisadores (B1), parece que as pesquisas investiram mais nas experiências narrativas dessas pessoas para avaliar serviços, tendo usuários entrevistando usuários.

Vale ressaltar que não encontramos nenhum artigo que tenha se aproximado de B2, e que quatro artigos usaram mais de um método para as avaliações.

Após esta apresentação descritiva dos dados quantitativos e bibliométricos, destacamos a análise qualitativa dos artigos mais recentes com métodos $A 1$, A2, B1 e os que utilizam mais de um método para a participação e avaliação dos usuários.

Tanto os Estados Unidos quanto a Inglaterra tiveram 4 artigos selecionados em A1. Destacamos um estudo(22) avaliativo de programas de saúde mental direcionados aos veteranos de guerra nos EUA, com método quantitativo tipo coorte, utilizando como ferramentas entrevistas por telefone, com instrumentos validados internacionalmente, com destaque para o RSA (recovery self-assessment). Os achados indicam que pessoas com comorbidades avaliaram melhor os atendimentos e tratamentos pelos programas, do que pessoas com quadros menos complexos. Contudo, no que diz respeito a práticas centradas na pessoa e para a recuperação pessoal, o programa teve uma avaliação inferior por todos os participantes em todas as faixas etárias, destacando assim o desafio desses programas em desenvolver melhorias nestes aspectos para garantir a qualidade de vida dos veteranos de guerra(22).

Encontramos três artigos brasileiros na categoria A1, nos anos de 2006, 209 e 2012. São estudos que trabalharam apenas com usuários, com instrumentos ou entrevistas fechadas, usando métodos de análise quantitativos, dois avaliando CAPS e um ambulatório. No geral, os usuários estavam satisfeitos com o tratamento e com os novos serviços prestados (23-25).

Todos os artigos por nós enquadrados no modelo A1 partiram de metodologias exclusivamente quantitativas e com análises correspondentes através de programas de mensuração dos dados(26-28). O que variou foram: as formas de coleta de dados: entrevistas de múltipla escolha ou aplicação de instrumentos com escalas tipo Likert(22,29-32); o tamanho da mostra e os participantes (com mais de 300 respondentes, entre trabalhadores, usuários e familiares)(27,33-35); a técnica de coleta de dados como: entrevistas feitas por telefone, instrumentos autoaplicáveis encaminhadas por cartas, correios eletrônicos ou dispostas na recepção dos serviços $^{(22,26,30,36)}$.

Esse formato de pesquisa avaliativa de serviços consegue captar e mensurar um maior número de dados, gerando uma análise mais geral de como os serviços têm prestado atendimento e como os atores envolvidos avaliam o tratamento e as consequências desse trabalho. Essas avaliações remetem as mensurações denominadas de $1^{\text {a }}$ geração avaliativa(37), na qual o avaliador é um técnico que deve saber construir e usar os instrumentos, e que qualquer variável da investigação/avaliação deve ser medida estatisticamente ${ }^{(37)}$.

$\mathrm{Na}$ categoria A2 destacamos um artigo sueco(38) que estudou apenas usuários com transtornos severos e persistentes, com base em entrevistas sobre moradias e o morar, o que costumamos chamar no Brasil de serviços residências terapêuticos (SRTs). O estudo de base fenomenológica buscou o consenso individual e coletivo sobre as narrativas produzidas, construindo um juízo de valor sobre o morar em SRTs. Foi possível destacar pontos positivos como: espaço de descanso, segurança e privacidade, com apoio de outros moradores e da equipe; mas também pontos negativos como: dependência, passividade e opressão por parte de moradores e equipe. O estudo conclui conjuntamente com os usuários que, para se ter uma moradia menos institucionalizada, o apoio deve direcionar-se à reabilitação e recuperação de $\operatorname{todos}^{(38)}$.

Os outros três artigos brasileiros encontram-se na categoria A2 ${ }^{(39-41)}$. Todos avaliaram os CAPS, a partir dos usuários e outros atores, utilizando grupos focais, entrevistas abertas e um usou a escala SATIS - BR(40). A análise dos dados foi feita com métodos quantitativos e qualitativos, sendo utilizado o ciclo hermenêutico no processo de coleta e interpretação dos $\operatorname{dados}^{(39,41)}$.

Nos três artigos os CAPS foram bem avaliados em relação às práticas terapêuticas empregadas, ao acolhimento a crise e o tratamento em liberdade. Pontos negativos são destacados pela falta de insumos, medições, infraestrutura precária dos prédios e dificuldade de integralidade com outros pontos da rede sócio assistencial. Refere-se a permanente avaliação dos serviços, como também à importância em fomentar processos de cidadania, participação social e dos familiares como rede de suporte e de cidadania, na garantia de direitos aos usuários ${ }^{(39-41)}$. 
Destacamos o trabalho(40) que avaliou um serviço tipo CAPS no Rio Grande do Sul, através de método qualitativo, com técnicas de grupos focais e análise hermenêutica na construção dos consensos. Participaram trabalhadores, usuários e familiares, que puderam discutir pontos positivos e desafios para os serviços, que tangem o poder contratual dos sujeitos perante seu tratamento no cotidiano.

$\mathrm{Na}$ categoria A2, a maioria dos artigos foi trabalhada a partir de métodos qualitativos com técnicas do tipo: entrevista semiestruturadas, narrativas e grupos focais(42). Apenas dois artigos partiram de métodos mistos ${ }^{(43-44)}$. Vale ressaltar que durante a análise dos dados os autores reforçam seus referenciais teóricos para tal, com destaques para a fenomenologia e o materialismo histórico, o que não encontramos nos artigos classificados como A1.

A categoria A2 pode se encaixar na avaliação de $2^{a}$ e $3^{a}$ gerações $^{(37)}$ : a descrição $\left(2^{a}\right)$, em que o foco é processo, e não somente na medição dos resultados; o julgamento $\left(3^{a}\right)$, em que o avaliador também julga e emite opiniões. Claramente, os artigos buscavam a experiência das pessoas com os serviços e os tratamentos recebidos e como os diferentes atores propunham mudanças no processo, mas as análises ainda se traduzem em narrativas interpretadas pelos pesquisadores $^{(37)}$.

Na categoria B1, nos chamaram a atenção os quatro artigos australianos ${ }^{(45-48)}$ mostrando outras formas de avaliação de serviço que encorajam maior participação dos atores envolvidos.

Um dos trabalhos( ${ }^{(45)}$ estudou a percepção dos usuários em relação aos serviços prestados em diferentes pontos da rede de saúde mental australiana, tendo como entrevistadores estudantes e usuários pesquisadores. As entrevistas eram semiestruturadas com foco na discussão sobre a percepção dos usuários em relação aos serviços. Em geral, os usuários destacaram a falta de informação durante o acesso aos serviços, principalmente nos hospitais, o que dificultava o empoderamento diante da situação vivida. Ainda em relação ao hospital, destacaram os efeitos negativos das medicações e o pouco diálogo com os médicos. 0 artigo também destacou que as avaliações de serviços de saúde devem ter o ponto de vista dos usuários na centralidade da discussão, para contribuir com práticas mais orientadas ao usuário e sua recuperação(45).

Outra diferença importante foi na forma como os usuários estavam envolvidos na pesquisa (pesquisadores entrevistadores), o que facilitou o acesso a outros usuários pesquisados, com relatos mais profundos durante as entrevistas do que às feitas pelos estudantes, indicando a pertinência dessa abordagem para avaliação de serviços. Este grupo de pesquisa defende que os usuários podem e devem participar de outros aspectos da pesquisa, buscando a profundidade do processo avaliativo, gerando dados mais fidedignos às realidades vivenciadas, incluindo questões realmente relevantes para os usuários ${ }^{(45-46)}$.

Os outros dois trabalhos ${ }^{(47-48)}$ discutem o processo avaliativo de serviços de atenção primária em saúde mental na Austrália, a partir de métodos qualitativos com usuários pesquisadores em grupos focais, entrevistas e construção de narrativas consensuadas. Os pontos fortes dos resultados indicaram para avaliação de tratamento recebido e terapêuticas de suporte e conexão social. Em relação à conexão social discutiu-se a importância do respeito e apoio de pares como ofertas dos serviços para a recuperação das pessoas. Para alguns usuários, o apoio de pares foi considerado o mais importante e fundamental para sua recuperação e boa avaliação dos serviços que indicaram ${ }^{(47-48)}$. Destacaram também, como importantes recursos para a recuperação e avaliação positivas dos serviços, o tratamento medicamentoso, planos e gerenciamento de crise e terapias espirituais ${ }^{(47-48)}$.

Os usuários discutiram e trouxeram avaliações negativas que merecem destaque: a falta de funcionários e de barreiras geográficas nos espaços rurais australianos, incapazes de acessar serviços, com demora de até 4 dias; falta de confiança nas equipes e a falta de sistematização na prática de apoio de pares nestas regiões(47-48). Já nos grandes centros, a queixa era a sensação de serem tratados como números e sintomas e não como pessoas singulares. Destacaram também a importância na ampliação da participação dos usuários nos processos de avaliação, desde o desenvolvimento até a análise de estratégias mais efetivas para a melhoria dos serviços ${ }^{(47-48)}$.

A categoria B1 talvez seja a que mais se aproxima de avaliações de $4^{a}$ geração(37) por meio dos pressupostos metodológicos do paradigma construtivista, em que as reivindicações, preocupações e questões dos grupos de interesse servem como foco organizacional da pesquisa e da informação necessária para as análises. Assim, os atores ou grupos de interesse estão envolvidos ou potencialmente afetados pelo serviço e pelas eventuais consequências do processo avaliativo(37).

Vale ressaltar que os usuários, quando participam de pesquisas, tendem a propor questões relevantes, identificar falhas na pesquisa, são bons indicadores da validade de determinados protocolos e instrumentos, oferecem interessantes interpretações e acompanham os resultados(49). No Brasil, a literatura sobre participação de usuários em pesquisas acadêmicas é limitada, sendo escassas e recentes as experiências de inserção de usuários em pesquisa(50).

Países como Austrália e Nova Zelândia incorporaram a participação de usuários da Saúde Mental em pesquisa 
enquanto direito dos mesmos. Pesquisas com relações mais horizontais entre pesquisadores acadêmicos e comunidades possibilitam o desenvolvimento profissional dos acadêmicos, tendem a ter maior credibilidade e envolvimento de usuários na adesão de outros pares para a pesquisa(51-52).

Em relação aos artigos sobre avaliações que utilizaram vários métodos e formas de inserção dos usuários no processo, destacamos um trabalho realizado na Inglaterra(53), que utilizou métodos quantitativos e análises estatísticas, como também qualitativos, com entrevistas, que foram realizadas por estudantes universitários e usuários do sistema, que haviam passado por experiências de internações. Os desafios levantados no processo avaliativo dos hospitais foram: deveres e disposições dos funcionários, ambiência e comunicação, principalmente relacionada às regras e menos a processos terapêuticos, como escuta acolhedora dos profissionais. Os usuários no presente estudo atribuíam grande importância à justiça, sendo ouvidos, tendo atividades e sendo tratados com respeito(53).

Os artigos com múltiplos métodos, com diferentes formas de participação dos usuários e outros atores aparecem em literatura mais recente, a partir de 2010, em países com forte tradição em avaliação de sistemas (Inglaterra e Canadá) ${ }^{(53-55)}$ e referências nas reformas do modelo assistencial em saúde mental (Itália) ${ }^{(56)}$.

Isto se apresenta para nós como um grande avanço na cultura avaliativa, buscando diminuir as distâncias entre modelos cartesianos quantitativos e modelos fenomenológicos, materialistas históricos e críticos. Consideramos fundamental o uso de múltiplos métodos, com a participação de maior número possível dos atores envolvidos, de diferentes formas, para uma avaliação de serviços realmente comprometida com a afirmação de práticas exitosas, como também para transformação de projetos que não servem ao público em questão.

Nos artigos internacionais, percebe-se maior número e investimento em processos avaliativos. Mesmo assim, a voz dos usuários aparece em menor número em relação aos trabalhadores e gestores. A grande maioria dos artigos avaliava apenas algumas condições ou práticas dos serviços e a satisfação em receber aquela atenção específica. Avaliações globais considerando o serviço em sua complexidade territorial e de rede, com múltiplas ofertas e com diversificação dos métodos e atores para a produção de resultados mais consistentes são restritas. Destacamos Inglaterra, Austrália, Canadá, EUA e Brasil com produções voltadas para avaliações mais globais.

Sabemos que há outros artigos sobre avaliação de serviços de saúde mental no Brasil, contudo nosso achado corrobora com outras revisões sobre a temática com diferentes objetivos ${ }^{(8,57-58)}$, no sentido que poucos estudos dão voz aos usuários e, quando o fazem, apenas avaliam algumas práticas e não o serviço como um todo.

\section{Limitações}

Esperávamos encontrar mais artigos internacionais valorizando avaliações globais com todos os atores envolvidos. O fato da nossa análise só contemplar produções que descrevessem avaliação dos serviços e não de práticas pontuais, como também as palavras-chave que os autores usaram nos artigos não contemplavam as usadas por nós na busca (saúde mental e avaliação), pode ter influenciado no nosso levantamento. Apesar de acreditarmos que os descritores foram bem abrangentes, podem não ter capturado algum artigo relevante tanto no panorama nacional, como no internacional.

\section{Conclusão}

Este artigo apresentou uma revisão sistemática qualitativa da literatura sobre avaliação de serviços da saúde mental, destacando as que incorporaram a participação dos usuários, identificando as publicações nacionais brasileiras e internacionais na área da saúde mental.

Os dados quantitativos encontrados nos mostraram que o Brasil tem pesquisas sobre avaliação de serviços de saúde mental, mas ainda em número menor, em comparação com outros países como Inglaterra, Austrália e EUA.

De acordo com esta revisão, a abordagem metodológica de incorporar a perspectiva do usuário, ou seja, a forma como os usuários são envolvidos nos estudos, tanto o Brasil quanto outros países, ainda é um desafio. Há poucos investimentos e esforços na construção de pesquisas compartilhadas e efetivas com o protagonismo dos usuários.

Considerando os resultados e a análise da nossa revisão, concluímos a importância em avançarmos em avaliações mais robustas dos serviços de saúde mental, que destacam a voz e a participação dos usuários, pois ainda são menor número no mundo; e no Brasil, coloca-se o desafio ainda de estruturar avaliações sistemáticas dos serviços públicos, com multiplicidade de métodos avaliativos e envolvendo todos os atores.

Consideramos que os estudos de revisão sistemática são sempre importantes instrumentos de reflexão para embasar pesquisas, iluminar fenômenos e demonstrar como outros pesquisadores têm estudado as temáticas.

\section{Referências}

1. Amarante P. Loucos pela vida: a trajetória da reforma psiquiátrica no Brasil. $3^{a}$ ed. Rio de Janeiro (RJ): Fiocruz; 1998. $136 \mathrm{p}$. 
2. Barros DD. Jardins de Abel: Desconstrução do Manicômio de Trieste. São Paulo (SP): Lemos; 1994. 158 p. 3. Mângia EF, Nicácio F. Terapia ocupacional em saúde mental: tendências principais e desafios contemporâneos. In: De Carlo MMRP, Bartalotti CC. organizadores. Terapia Ocupacional no Brasil: fundamentos e perspectivas. São Paulo (SP): Plexus; 2001. p. 63-80.

4. Lancetti A, Amarante P. Saúde mental e saúde coletiva. In: Campos GWS, Minayo MC, Akerman M, Drumond M, Carvalho YM, organizadores. Tratado de Saúde Coletiva. São Paulo (SP): Hucitec; 2006. p. 615-34.

5. Ministério da Saúde. Reforma Psiquiátrica e política de Saúde Mental no Brasil [Internet]. Brasília (DF): Ministério da Saúde; 2005. [Acesso 16 ago 2018]. Disponível em: http://bvsms.saude.gov.br/bvs/ publicacoes/Relatorio15_anos_Caracas.pdf

6. Lei Federal №. 10216, de 6 de abril de 2001 (BR). Proteção e os direitos das pessoas portadoras de transtornos mentais e redireciona o modelo assistencial em saúde mental. [Internet]. Brasília (DF): Ministério da Saúde; 2001. [Acesso 16 ago 2018]. Disponível em: http:// www.planalto.gov.br/ccivil_03/leis/leis_2001/l10216.htm

7. Trapé TL. Rede de atenção à saúde mental: Estudo comparado Brasil - Catalunha [Internet]. [tese]. Campinas (SP): Universidade Estadual de Campinas; 2015. [Acesso 16 ago 2018]. Disponível em: http:// repositorio.unicamp.br/handle/REPOSIP/313041

8. Dantas C de R, Oda AMGR. Cartografia das pesquisas avaliativas de serviços de saúde mental no Brasil (2004-2013). Physis. [Internet]. 2014; 24(4):1127-79. [Acesso 23 Jan 2018];Disponívelem:http://www.scielo.br/scielo.php? script=sci_arttext\&pid=S0103-73312014000401127\&lng= pt\&tlng $=p t$

9. WHO. Mental Health - Action Plan 2013-2020 [Internet]. Geneva (SW); World Health Organization; 2013. [Acesso Jan 10 2018]. Available at: http://apps.who.int/iris/ bitstream/10665/89966/1/9789241506021_eng.pdf?ua=1 10. WHO. Atlas Mental Health [Internet]. Geneva (SW); World Health Organization; 2014. [cited Jan 10 2018]. Available at: http://www.who.int/mental_health/

11. Dimov T, Ricci EC. A pesquisa acadêmica como atividade humana: participação de usuários da saúde mental e as contribuições da terapia ocupacional. Cad Ter Ocup UFSCar. [Internet]. 2016;24(3):651-8. [Acesso 23 jan 2018]. Disponível em: http://doi.editoracubo.com. br/10.4322/0104-4931.ctoEN0674

12. Onocko Campos R. Clínica: a palavra negada - sobre as práticas clínicas nos serviços substitutivos de Saúde Mental. Saúde em Debate. [Internet]. 2000;25(58):98-111. [Acesso 14 fev 2018]. Disponível em: http://www.ensp. fiocruz.br/parcerias/cebes/cebes.html

13. Scriven M. Metodologia da Avaliação. In: Instituto Fonte, organizador. Introdução à avaliação de programas sociais. São Paulo (SP): Instituto Fonte; 2003. p. 8-33.
14. Furtado JP. Avaliação de Programas e Serviços. In: Campos GWS, Minayo MC, Akerman M, Drumond, M, Carvalho, YM, organizadores. Tratado de saúde coletiva. $2^{\circ}$ ed São Paulo (SP): Hucitec; 2009. p. 715-39.

15. Liberati A, Altman DG, Tetzlaff J, Mulrow C, Gøtzsche PC, Ioannidis JPA, et al. The PRISMA statement for reporting systematic reviews and meta-analyses of studies that evaluate health care interventions: explanation and elaboration. PLoS Med. [Internet]. 2009;6(7). [cited Nov 1 2017]. Available from: https://journals.plos.org/ plosmedicine/article?id=10.1371/journal. pmed. 1000100 16. Moher D, Liberati A, Tetzlaff J, Altman DG, Group TP. Preferred Reporting Items for Systematic Reviews and Meta-Analyses: The PRISMA Statement. PLoS Med. [Internet]. 2009;6(7). [cited Nov 1 2017]. Available from: http://dx.plos.org/10.1371/journal.pmed.1000097 17. Lopes ALM, Fracolli LA. Revisão sistemática de literatura e metassíntese qualitativa: considerações sobre sua aplicação na pesquisa em enfermagem. Texto Contexto Enferm. [Internet]. 2008;17(4):771-8. [Acesso 21 nov 2017]. Disponível em: http://www. redalyc.org/articulo.oa?id $=71411240019$

18. Hutton B, Salanti G, Caldwell DM, Chaimani A, Schmid CH, Cameron $C$, et al The PRISMA extension statement for reporting of systematic reviews incorporating network meta-analyses of health care interventions: Checklist and explanations. Ann Intern Med. [Internet]. 2015;162(11):777-84. [cited Nov 21 2017]. Available from: http://annals.org/aim/fullarticle/2299856

19. Moher D, Liberati A, Tetzlaff J, Altman D. Principais itens para relatar Revisões sistemáticas e Meta-análises: A recomendação PRISMA*. Epidemiol Serv Saude. [Internet]. 2015;24(2):335-42. [Acesso 16 ago 2017]. Disponível em: www.prisma-statement.org.

20. Rose D. Having a diagnosis is a qualification for the job. BMJ. [Internet]. 2003;326(7402):1331. [cited Feb 4 2018]. Available from: http://www.ncbi.nlm.nih.gov/pubmed/ 12805192

21. Campos GW de S. Produção de Conhecimento, avaliação de políticas públicas em saúde mental:notas reflexivas. In: Onocko-Campos R, Furtado JP, Passos E, Benevides R, organizadores. Pesquisa Avaliativa em Saúde Mental - desenho participativo e efeitos da narratividade. Hucite: São Paulo (SP): Aderaldo \& Rothschild Editores; 2008. p. 97-102.

22. Blonigen DM, Bui L, Harris AHS, Hepner KA, Kivlahan DR. Perceptions of behavioral health care among veterans with substance use disorders: Results from a national evaluation of mental health services in the Veterans Health Administration. J Subst Abuse Treat. [Internet]. 2014;47(2):122-9. [cited Aug 31 2018]. Available from: https://www.sciencedirect.com/science/ article/pii/S0740547214000725

23. Silva MA da, Bandeira M, Scalon JD, Quaglia MAC. Satisfação dos pacientes com os serviços de saúde mental: 
a percepção de mudanças como preditora. J Bras Psiquiatr. [Internet]. 2012;61(2):64-71. [Acesso 4 set 2018]. Disponível em: http://www.scielo.br/scielo.php? script $=$ sci_arttext\&pid $=$ S0047-20852012000200 $002 \& \operatorname{lng}=p t \& t \operatorname{lng}=p t$

24. Heckert U, Teixeira LS, Trindade A de S. Avaliação da satisfação dos usuários do Centro Regional de Referência em Saúde Mental (CRRESAM) da região central de Juiz de Fora, MG. HU Rev. [Internet]. 2006;32(1):15-20. [Acesso 4 set 2018]. Disponível em: https://hurevista. ufjf.emnuvens.com.br/hurevista/article/view/6/3

25. Tomasi E, Facchini LA, Piccini RX, Thumé E, Silva $\mathrm{RA}$ da, Gonçalves $\mathrm{H}$, et al. Efetividade dos centros de atenção psicossocial no cuidado a portadores de sofrimento psíquico em cidade de porte médio do Sul do Brasil: uma análise estratificada. Cad Saúde Pública. [Internet]. 2010;26(4):807-15. [Acesso 4 set 2018]. Disponível em: http://www.scielo.br/scielo. php?script $=$ sci_arttext $\&$ pid $=$ S0102-311X20100004000 22\&lng=pt\&tIng=pt

26. Shamir D, Szor H, Melamed Y. Dropout, early termination and detachment from a public psychiatric clinic. Psychiatr Danub. [Internet]. 2010;22(1):46-50. [cited Aug 31 2018] Available from: https://hrcak.srce. hr/index.php?show=clanak\&id_clanak_jezik $=74997$

27. Whittington R, Bowers L, Nolan P, Simpson A, Neil L. Approval Ratings of Inpatient Coercive Interventions in a National Sample of Mental Health Service Users and Staff in England. Psychiatr Serv [Internet]. 2009;60(6):792-8. [cited Aug 31 2018]. Available from: http:// psychiatryonline.org/doi/abs/10.1176/ps.2009.60.6.792 28. Remnik Y, Melamed Y, Swartz M, Elizur A, Barak Y. Patients' Satisfaction with Psychiatric Inpatient Care. Isr J Psychiatry Relat Sci. [Internet]. 2004;41(3):208-12. [cited Aug 31 2018]. Available from: https://search. proquest.com/docview/236928012/fulltextPDF/C6E7A7 88E3054D36PQ/1 ?accountid $=8113$

29. Ruggeri M, Salvi G, Perwanger V, Phelan M, Pellegrini N, Parabiaghi A. Satisfaction with community and hospital-based emergency services amongst severely mentally ill service users. Soc Psychiatry Psychiatr Epidemiol. [Internet]. 2006;41(4):302-9. [cited Aug 31 2018]. Available at: http://link.springer.com/10.1007/ s00127-006-0030-x

30. Mavrogiorgou $P$, Siebers $F$, Juckel G, Kienast $T$. Patient satisfaction with specialized mental health service for obsessive-compulsive disorder. Ann Gen Psychiatry. [Internet]. 2013;12(1):41. [cited Aug 31 2018]. Available from: http://annals-general-psychiatry. biomedcentral.com/articles/10.1186/1744-859X-12-41 31. Lundqvist L-O, Ahlström G, Wilde-Larsson B, Schröder A. The patient's view of quality in psychiatric outpatient care. J Psychiatr Ment Health Nurs. [Internet]. 2012;19(7):629-37 [cited Aug 31 2018]. Available from: http://doi. wiley.com/10.1111/j.1365-2850.2012.01899.x
32. Tierney KR, Kane CF. Promoting Wellness and Recovery for Persons With Serious Mental Illness: A Program Evaluation. Arch Psychiatr Nurs. [Internet]. 2011;25(2):77-89. [cited Aug 31 2018]. Available from: https://www.sciencedirect.com/science/article/ pii/S0883941710000853

33. Day CA, Demirkol A, Tynan M, Curry K, Hines S, Lintzeris $N$, et al. Individual versus team-based case-management for clients of opioid treatment services: An initial evaluation of what clients prefer. Drug Alcohol Rev. [Internet]. 2012;31(4):499-506. [cited Aug 31 2018]. Available from: http://doi.wiley. com/10.1111/j.1465-3362.2011.00347.x

34. Gould MS, Munfakh JLH, Kleinman M, Lake AM. National Suicide Prevention Lifeline: Enhancing Mental Health Care for Suicidal Individuals and Other People in Crisis. Suicide Life Threat Behav. [Internet]. 2012;42(1):22-35 [cited Aug 31 2018]. Available from: http://doi.wiley.com/10.1111/j.1943-278X.2011.00068.x 35. Solomon $\mathrm{P}$, Stanhope V. Recovery: Expanding the Vision of Evidence-Based Practice. $\mathrm{Br}$ Treat Cris Interv. [Internet]. 2004;4:311-21 [cited Jan 17 2018]. Available from:https://www.researchgate.net/profile/ Victoria_Stanhope/publication/31362986_Recovery_ Expanding_the_Vision_of_Evidence-Based_Practice/ links/02e7e525ed47e049f9000000.pdf

36. Hill S, Turner N, Barry S, O'Callaghan E. Client satisfaction among outpatients attending an Irish community mental health service. Ir J Psychol Med. [Internet]. 2009;26(03):127-30. [cited Aug 31 2018]. Available from: https://www.cambridge.org/ core/product/identifier/S0790966700000422/type/ journal_article

37. Guba EG, Lincoln YS. Fourth generation evaluation [Internet]. SAGE Publications, organizador. United States of America (USA): Sage Publications; 1989. 294 p. [cited Sep 1 2018]. Available from: https://books.google.com. $\mathrm{br} /$ books? $\mathrm{hl}=$ en\&lr=\&id=k_zXEUst46UC\&oi=fnd\&pg $=P$ A7\&dq $=$ GUBA,$+E . \% 3 B+$ LINCOLN,$+Y$. +Effective+evalu tion.\&ots $=\_5 a v n h T E Z \& s i g=X T F w t Z u j R r D 5 \times n 9 Q f b i j R 9$ jIbWE\&redir_esc $=y \# v=$ onepage $\& q=G U B A \% 2 C$ E. \%3B LINCOLN\%2C $Y$. Effective evalution. \& $\mathrm{f}=\mathrm{f}$

38. Bengtsson-Tops A, Ericsson U, Ehliasson K. Living in supportive housing for people with serious mental illness: A paradoxical everyday life. Int J Ment Health Nurs. [Internet]. 2014;23(5):409-18. [cited Aug 31 2018]. Available from: http://doi.wiley.com/10.1111/ inm. 12072

39. Schneider JF, Wagner M, Prado L. Avaliação de um centro de atenção psicossocial brasileiro. Cienc y Enferm. [Internet]. 2009;15(3):91-100 [citado 04 Sep 2018]. Disponível em: https://scielo.conicyt.cl/pdf/cienf/v15n3/ art_10.pdf

40. Kantorski LP, Jardim V da R, Wetzel C, Olschowsky A, Schneider JF, Heck RM, et al. Satisfação dos usuários dos 
centros de atenção psicossocial da região Sul do Brasil. Rev Saúde Pública. [Internet]. 2009;43(1):29-35. [Acesso 4 set 2018]. Disponível em: https://www. scielosp.org/pdf/rsp/2009.v43suppl1/29-35/pt

41. Surjus L, Onocko-Campos R. A avaliação dos usuários sobre os Centros de Atenção Psicossocial (CAPS) de Campinas, SP. Rev Latinoam Psicopatol Fundam. [Internet]. 2011;14(1):122-33. [Acesso 4 set 2018]. Disponível em: http://www.redalyc.org/html/2330/233018495009/

42. Restall G, Strutt C. Participation in planning and evaluating mental health services: Building capacity. Psychiatr Rehabil J. [Internet]. 2008;31(3):234-8. [cited Aug 31 2018]. Available from: http://doi.apa.org/ getdoi.cfm?doi=10.2975/31.3.2008.234.238

43. Rogers A, Oliver D, Bower P, Lovell K, Richards D. Peoples' understandings of a primary care-based mental health self-help clinic. Patient Educ Couns. [Internet]. 2004;53(1):41-6. [cited Aug 31 2018]. Available from: https://www.sciencedirect.com/science/article/pii/ S0738399103001149

44. Hodge S, Barr W, Göpfert M, Hellin K, Horne A, Kirkcaldy A. Qualitative findings from a mixed methods evaluation of once-weekly therapeutic community day services for people with personality disorder. J Ment Heal. [Internet]. 2010;19(1):43-51. [cited Aug 31 2018]. Available from: http://www.tandfonline.com/doi/ full/10.3109/09638230903469152

45. Malins G, Oades L, Viney L, Aspden S. What's in a service? Consumers' views of Australian mental health services. Psychiatr Rehabil J. [Internet]. 2006;29(3):197-204. [cited Aug 31 2018]. Available from: http://doi.apa.org/getdoi. cfm?doi=10.2975/29.2006.197.204

46. Happell B, Roper C. Consumer Participation in Mental Health Research: Articulating a Model to Guide Practice. Australas Psychiatry. [Internet]. 2007;15(3):237-41. [cited Feb 4 2018]. Available from: http://journals. sagepub.com/doi/10.1080/10398560701320113

47. Happell B. Determining the effectiveness of mental health services from a consumer perspective: Part 1: Enhancing recovery. Int J Ment Health Nurs. [Internet]. 2008;17(2):116-22. [cited Feb 4 2018]. Available from: https://onlinelibrary.wiley.com/doi/ abs/10.1111/j.1447-0349.2008.00519.x

48. Happell B. Determining the effectiveness of mental health services from a consumer perspective: Part 2: Barriers to recovery and principles for evaluation. Int $]$ Ment Health Nurs. [Internet]. 2008;17(2):123-30. [cited Feb 4 2018]. Available from: https://onlinelibrary.wiley. com/doi/abs/10.1111/j.1447-0349.2008.00520.x

49. Ning L, Weavell W, Woodhouse S. Consumer participation in research. Psychiatric Disability Services of Victoria. Victoria; 2010. [cited Sep 21 2017]. Available from: http://mheco.org.au/attachments/NewParadigm_ Au-tumn2010_Consumer_Research.pdf
50. Furtado JP, Campos RO. Participação, produção de conhecimento e pesquisa avaliativa: a inserção de diferentes atores em uma investigação em saúde mental. Cad Saúde Pública. [Internet]. 2008;24(11):2671-80. [Acesso 5 set 2018]. Disponível em: http://www.scielo. br/scielo.php?script=sci_arttext\&pid=S0102-311X2008 001100022\&lng=pt\&tIng=pt

51. Presotto RF. Participação de Usuários de Serviços de Saúde Mental em Pesquisas: Um olhar a partir dos conceitos de Empowerment e Recovery [Internet]. [Dissertação] Campinas (SP). Universidade Estadual de Campinas; 2013. [Acesso 12 out 2017]. Disponível em: http://repositorio.unicamp.br/bitstream/ REPOSIP/311879/1/Presotto_RodrigoFernando_M.pdf

52. Nascimento NS do, Nogueira A, Presotto RF, Angelini CR, Enes G da ST, Bilbao EV, et al. Cad Bras Saúde Mental. [Internet]. 2017;9(01):271-81. [Acesso 4 Fev 2018]. Disponível em: http://stat.ijkem.incubadora.ufsc. br/index.php/cbsm/article/view/4691

53. Stewart D, Burrow H, Duckworth A, Dhillon J, Fife S, Kelly $S$, et al. Thematic analysis of psychiatric patients' perceptions of nursing staff. Int J Ment Health Nurs. [Internet]. 2015;24(1):82-90. [cited Aug 31 2018]. Available at: http://doi.wiley.com/10.1111/inm.12107 54. Taylor S, Abbott S, Hardy S. The INFORM Project: A Service User-Led Research Endeavor. Arch Psychiatr Nurs. [Internet]. 2012;26(6):448-56. [cited Aug 31 2018]. Available from: https://www.sciencedirect.com/ science/article/pii/S0883941712000337

55. Tempier R, Hepp SL, Duncan CR, Rohr B, Hachey K, Mosier K. Patient-Centered Care in Affective, NonAffective, and Schizoaffective Groups: Patients' Opinions and Attitudes. Commun Ment Health J. [Internet]. 2010;46(5):452-60. [cited Aug 31 2018]. Available from: http://link.springer.com/10.1007/ s10597-010-9316-2

56. Barbato A, Bajoni A, Rapisarda F, D'Anza V, De Luca LF, Inglese $C$, et al. Quality Assessment of Mental Health Care by People with Severe Mental Disorders: A Participatory Research Project. Community Ment Health J. [Internet]. 2014;50(4):402-8. [cited Aug 31 2018]. Available from: http://link.springer.com/10.1007/ s10597-013-9667-6

57. Wetzel C, Kantorski LP. Avaliação de serviços em saúde mental no contexto da reforma psiquiátrica. Texto Contexto Enferm. [Internet]. 2004;13(4):593-8. [Acesso 4 set 2018]. Disponível em: http://www.redalyc. org/html/714/71413412/

58. Silva N dos S, Melo JM, Esperidião E. Avaliação dos serviços de assistência em saúde mental brasileiros: revisão integrativa da literatura. Rev Min Enferm. [Internet]. 2012;16(2):280-8. [Acesso 5 set 2018]. Disponível em: http://www.reme.org.br/artigo/ detalhes/530 


\section{Contribuição dos autores}

Concepção e desenho da pesquisa: Éllen Cristina Ricci, Mariana Barbosa Pereira, Leidy Janeth Erazo, Rosana Teresa Onocko-Campos e Erotildes Maria Leal. Obtenção de dados: Éllen Cristina Ricci, Mariana Barbosa Pereira e Leidy Janeth Erazo. Análise e interpretação dos dados: Éllen Cristina Ricci, Mariana Barbosa Pereira, Leidy Janeth Erazo e Erotildes Maria Leal. Redação do manuscrito: Éllen Cristina Ricci, Mariana Barbosa Pereira, Leidy Janeth Erazo, Rosana Teresa Onocko-Campos e Erotildes Maria Leal. Revisão crítica do manuscrito quanto ao conteúdo intelectual importante Éllen Cristina Ricci, Mariana Barbosa Pereira, Leidy Janeth Erazo, Rosana Teresa Onocko-Campos e Erotildes Maria Leal.

Todos os autores aprovaram a versão final do texto.

Conflito de interesse: os autores declararam que não há conflito de interesse.

Esta licença permite que outros remixem, adaptem e criem a partir do seu trabalho para fins não comerciais, e embora os novos trabalhos tenham de lhe atribuir o devido crédito e não possam ser usados para fins comerciais, os usuários não têm de licenciar esses trabalhos derivados sob os mesmos termos. 